\title{
Trayectorias sanitarias durante 4 años de niños expuestos prenatalmente a cocaína y/o cannabis. Estudio de cohorte retrospectivo en La Pampa, Argentina \\ Four-year health trajectories of children prenatally exposed to cocaine and/or cannabis. A retrospective, cohort study in $\mathrm{La}$ Pampa, Argentina
}

Mg. Méd. Marina Villarreal ${ }^{a, b}$, Mg. Valeria Belmonte ${ }^{b}$, Méd. Agustina Abdala $^{a}$ y Dr. Jorge L. Olivares. ${ }^{a, b}$

\section{RESUMEN}

El consumo de cocaína y / o cannabis durante el embarazo constituye un problema en ascenso, de importancia para la salud pública mundial. Los niños expuestos pueden presentar un amplio rango de complicaciones en el período perinatal, pero los conocimientos sobre la evolución posterior son escasos.

Objetivo: Describir y comparar las trayectorias sanitarias de niños expuestos y no expuestos prenatalmente a cocaína y/o cannabis durante 4 años.

Métodos: Estudio de cohorte retrospectivo con grupo de comparación doble. Los niños expuestos fueron detectados en el Servicio de Neonatología de un hospital público mediante una prueba de orina, entre 2009 y 2013.

a. Establecimiento Asistencial Dr. Lucio Molas (E. A. L. Molas), Santa Rosa, La Pampa.

b. Universidad Nacional de La Pampa.

Correspondencia: Mg. Méd. Marina Villarreal: marvillarreal@cpenet. com.ar

\section{Financiamiento:} Beca Salud Investiga 2017 "Dr. Abraam Sonis", Ministerio de Salud de la Nación, Dirección de Investigación para la Salud.

Conflicto de intereses: Ninguno que declarar.

Recibido: 2-7-2018 Aceptado: 12-5-2019
Resultados:Se evaluaron 29 niños expuestos y 58 no expuestos. Las principales drogas detectadas enelgrupoexpuestofueron cocaína y cannabis. La mayoría de las madres fueron policonsumidoras. En los niños del grupo expuesto, se encontraron diferencias significativas en menor frecuencia de controles desalud $(\mathrm{p}<0,0001)$ y mayor frecuencia de consultas en Emergencias $(\mathrm{p}=0,0295)$ e Internaciones $(p=0,007)$, principalmente, por cuadros respiratorios. Presentaron, además, mayor frecuencia de enfermedad pulmonar obstructiva crónica, cambios de hogar y judicialización. En ese grupo, hubo 1 niño y 2 progenitores muertos por causa violenta. No hubo ninguna muerte en el grupo no expuesto. Conclusiones: Los niños expuestos a cocaína $\mathrm{y} / \mathrm{o}$ cannabis prenatalmente tuvieron menor número de controles de salud y mayor frecuencia de consultas en Emergencias e Internaciones. Presentaron, además, mayor frecuencia de enfermedad pulmonar obstructiva crónica, cambios de hogar, judicialización y muertes violentas en el grupo familiar directo.

Palabras clave: efectos tardíos en la exposición prenatal, cocaina, cannabis, seguimiento, morbimortalidad.

http:/ / dx.doi.org/10.5546/ aap.2019.360

Texto completo en inglés:

http:/ / dx.doi.org/10.5546/ aap.2019.eng.360
Cómo citar: Villarreal M, Belmonte V, Abdala A, Olivares JL. Trayectorias sanitarias durante 4 años de niños expuestos prenatalmente a cocaína y/o cannabis. Estudio de cohorte retrospectivo en La Pampa, Argentina. Arch Argent Pediatr 2019;117(6):360-367.

\section{INTRODUCCIÓN}

En la mayoría de los países del mundo, en la actualidad, el consumo de cannabis y cocaína durante el embarazo está en aumento, y el policonsumo es el patrón de uso más común. . $^{1-8}$

En el Servicio de Neonatología del hospital público en que se realizó este estudio, cocaína y cannabis fueron las drogas halladas con mayor frecuencia desde 2009, año en que se inició la detección en pruebas de orina posparto. $^{6}$

En términos generales, los niños expuestos prenatalmente a cannabis y / o cocaína pueden presentar un amplio rango de complicaciones, principalmente estudiadas durante el período perinatal..$^{2,49-19} \mathrm{En}$ la infancia, se describen consecuencias adversas en el corto y largo plazo, en especial, comprobadas en algunos aspectos del neurodesarrollo. ${ }^{12-16}$

En relación con las trayectorias sanitarias de estos niños, los datos en la literatura son escasos. En un estudio chileno, se observó que 100 niños con exposición prenatal a cocaína y otras drogas presentaron frecuentes inasistencias a controles y estudios, y reiteradas internaciones. ${ }^{18}$ 
Se ha descrito que los niños expuestos a drogas intraútero tuvieron un mayor número de consultas por lesiones compatibles con abuso y negligencia comparados con niños de la población general, con elevados porcentajes de intervenciones de servicios sociales y/o judiciales. En la mayoría de los casos, los padres biológicos fueron los causantes del maltrato, y ésta fue la principal causa de retirada permanente o temporaria de los niños de sus hogares. ${ }^{8,9,13,18,19}$

Además de la morbilidad referida, algunos autores han descrito mayor mortalidad en los niños expuestos intraútero que en los no expuestos, pero solo cuando se detectaron cocaína y opiáceos, y cuando el peso al nacer fue menor de 2500 g. $^{20}$

Los objetivos del estudio fueron describir y analizar las instancias de contacto con el sistema de salud registradas durante los primeros cuatro años de vida de los niños expuestos prenatalmente a cocaína y/o cannabis, comparándolos con los niños no expuestos. Secundariamente, describir las enfermedades adquiridas, la situación familiar/legal y la mortalidad de los niños o del grupo familiar directo.

\section{POBLACIÓN Y MÉTODOS \\ Estudio de cohorte retrospectivo}

Niños expuestos (grupo expuesto -GE-): niños nacidos entre 2009 y 2013 en el Servicio de Neonatología del Establecimiento Asistencial Dr. Lucio Molas de La Pampa, con detección de cocaína y/o cannabis en una muestra de orina posparto (de la madre y/o del niño). La muestra se solicitó ante la presencia de criterios de detección (Tabla 1).

Niños no expuestos (grupo de comparación): niños sin criterios de detección, nacidos en el mismo Servicio de Neonatología el mismo día o semana que los expuestos. Dos investigadores revisaron los libros de nacimientos hasta encontrar a los recién nacidos que más se asemejaran a cada nacido expuesto, considerando por orden de prioridad la edad materna, el peso al nacer, la edad gestacional, la situación ante la seguridad social y la localidad de procedencia.

Se seleccionaron 2 niños no expuestos por cada niño expuesto para aumentar la potencia del estudio. No hubo criterios de exclusión.

Las variables de estudio se obtuvieron de las siguientes fuentes secundarias (para algunas, se utilizaron ambas):

- Del Sistema Informático de Salud (SIS) de La Pampa: datos socioeconómicos, consultas, vacunas e internaciones, enfermedades adquiridas. En esta base, se encontraban los datos demográficos y una historia clínica resumida de los individuos asistidos en todos los establecimientos públicos de salud de la provincia. Los diagnósticos estaban registrados según la Clasificación Internacional de Enfermedades (CIE-10).

- De las historias clínicas del Hospital: datos socioeconómicos, perinatales y obstétricos, situación familiar/legal, internaciones, enfermedades adquiridas, mortalidad del niño y/o del núcleo familiar. El Molas es el Hospital público de mayor complejidad de Santa Rosa y de referencia por ser una de las pocas instituciones de salud de la provincia que contaba con servicio, especialidades y guardia activa pediátrica.

El estudio se realizó desde 6/2017 hasta $6 / 2018$. Semanalmente, 2 investigadores obtuvieron los datos de los niños del SIS, y otros 2 revisaron historias clínicas (20 pacientes por mes), y completaron una base de datos anonimizada diseñada para el estudio.

El estudio fue autorizado por el Comité de Ética en Investigación del Hospital Molas. La confidencialidad se mantuvo codificando y limitando la búsqueda, registro, análisis y acceso a la base de datos del estudio a los 4 autores / investigadores. No se registraron iniciales, nombres ni otros datos identificatorios de pacientes.

Para la carga, el procesamiento inicial y las presentaciones gráficas de datos, se utilizó

TABLA 1. Criterios de selección para realizar la prueba de detección de drogas ilícitas en la orina. Servicio de Neonatología, Hospital Molas, La Pampa. De 2009 a 2018

1. Comunicación actual o historia de uso de drogas.

2. Estado mental alterado en la madre.

3. Ausencia de control prenatal.

4. Complicaciones del sistema nervioso central inexplicables en el recién nacido.

5. Síntomas compatibles con abstinencia a drogas en el recién nacido.

6. Cambios en la conducta neonatal. 
Excel de Office 4.0. Para el análisis estadístico, el programa InfoStat, Universidad de Córdoba. La caracterización de los niños se realizó por medio de distribuciones de frecuencias absolutas y relativas para variables cualitativas, y medidas de centralización, dispersión e intervalos de confianza para variables cuantitativas. Para los análisis comparativos, se utilizaron pruebas de $\mathrm{chi}^{2}, t$ de Student y prueba de proporciones. Se consideró diferencia estadísticamente significativa con $\mathrm{p}<0,05$.

\section{RESULTADOS}

El GE estuvo constituido por 29 niños, y el grupo no expuesto (GNE), por 58. En el primero, 16 niños (el $55 \%$ ) fueron de sexo masculino; en el GNE, 34 (el $59 \%$ ).

Las comparaciones de los grupos según las variables de selección se presentan en la Tabla 2, donde puede observarse que son homogéneos.

En ambos grupos, más del $95 \%$ de las madres residían en el conglomerado geográfico Santa Rosa-Toay, de la provincia de La Pampa. Todos fueron pacientes asistidos al nacer en el mismo hospital público y sin obra social.

Las drogas detectadas en orina en el GE, en la madre y/o en el hijo, fueron solo cocaína en 9 (el $31 \%$ ), solo tetrahidrocannabinol en 10 (el $35 \%$ ), cocaína y tetrahidrocannabinol en 5 (el $17 \%$ ), cocaína con fenciclidina y/o benzodiacepina en $5(\mathrm{el} 17 \%)$.

La mayoría de las madres de los niños del GE fueron policonsumidoras durante el embarazo. Además de las drogas detectadas en la orina, reconocieron haber consumido alcohol (el $55 \%$ ) y/o tabaco (el $93 \%$ ). Ninguna mujer del GNE había consumido alcohol, y el $21 \%$ había consumido tabaco. Las diferencias entre grupos para el consumo de esas sustancias fueron significativas $(p<0,0001)$.
Pueden observarse, en la Tabla 3, datos socioeconómicos, familiares y obstétricos de las madres de los niños. En la Tabla 4, se presentan resultados perinatales.

Al egreso de Neonatología, ningún niño del GE presentaba patología. Dos niños del GNE tenían una cardiopatía congénita acianótica, sin requerimiento de medicación. Durante los 4 años del estudio, no hubo pérdidas de seguimiento.

\section{Consultas, vacunación e internaciones}

Los niños del GE fueron llevados a menos controles de salud, pero a más consultas de emergencias que los niños del GNE (Tabla 5 ).

El $93 \%$ del GE tuvo, al menos, 1 consulta en Emergencias, comparado con el $79 \%$ del GNE. Los principales motivos de consulta en Emergencias fueron respiratorios, en el $55 \%$ del GE y en el $26 \%$ del GNE ( $p<0,001)$. A los 4 años, todos los niños de ambos grupos tenían vacunación completa.

Fueron internados en un Servicio de Pediatría 19/29 niños (el $66 \%$ ) del GE, en comparación con $20 / 58$ (el $35 \%$ ) del GNE ( $\mathrm{p}=0,007)$. Las internaciones fueron 40 en el GE y 23 en el GNE. Los motivos de internación se muestran en la Figura 1.

Durante los 4 años de estudio, 14 niños (el $48 \%$ ) del GE desarrollaron enfermedad pulmonar obstructiva crónica, 9/14 codificados como J45 ("asma") y 5/14, como J44 ("otras enfermedades obstructivas crónicas"). No hubo casos en el GNE.

\section{Mortalidad}

En el GE, hubo 3 muertes violentas en 3 familias diferentes durante los 4 años del estudio. Una madre falleció en un siniestro vial al manejar alcoholizada. Un padre falleció por una herida de arma blanca, y un niño, por lesiones causadas por un arma de fuego, ambos en sus hogares. No hubo fallecimientos de niños ni de progenitores en el GNE.

Tabla 2. Comparación del grupo expuesto y no expuesto, según las variables de selección. Nacidos en el Hospital Molas, La Pampa, entre 2009 y 2013

\begin{tabular}{llccc}
\hline & & $\begin{array}{c}\text { Grupo expuesto } \\
(\mathbf{n}=\mathbf{2 9})\end{array}$ & $\begin{array}{c}\text { Grupo no expuesto } \\
(\mathbf{n}=\mathbf{5 8})\end{array}$ & $\begin{array}{c}\text { Comparación } \\
\text { entre grupos }\end{array}$ \\
\hline Peso del niño al nacer (gramos) & Media (DE) & $3132(670)$ & $3355(444)$ & \\
& IC 95\% & $2877-3387$ & $3265-3446$ & $\mathrm{p}=0,10$ \\
Edad gestacional del niño (semanas) & Media (DE) & $39(2)$ & $39(1)$ & $\mathrm{p}=0,25$ \\
& IC 95\% & $38-39$ & $39-40$ & $\mathrm{p}=0,64$ \\
\hline
\end{tabular}




\section{Situación familiar/legal}

En el GE, 8 niños (el $28 \%$ ) cambiaron de hogar (de vivienda y de adulto a cargo) durante sus 4 primeros años de vida (sin considerar el cambio realizado por intervención judicial en algunos casos, que sucedió posteriormente). De esos 8 niños, 3 cambiaron de hogar 2 veces, y 1, 5 veces. Esto significó ir a vivir con algún familiar diferente de los padres biológicos o con amigos o vecinos de algún progenitor.

Alguno de los padres biológicos estuvo privado de su libertad durante los 4 años del estudio en 8 niños del GE (el $28 \%$ ). No hubo padres en esa condición en el GNE.

En el GE, 8 niños (el $28 \%$ ) tuvieron solicitud de intervención judicial desde el sistema de salud. Se trató de 4 casos de maltrato físico: en 2, la madre había entregado al niño a un adulto no familiar; en 1, la madre estaba privada de su libertad; y, en otro, no se encontraron datos. El rango de edad al momento de la judicialización fue de 2 meses a 3 años de vida. En el GNE, no hubo ninguna solicitud de judicialización.

A los 4 años de vida, 17 niños del GE convivían con su madre (el $59 \%$ ); 7 (el $24 \%$ ), con una abuela; 3 , con padres sustitutos; 1 , con padres adoptivos; y 1, sin datos. En el GNE, todos vivían con algún padre biológico.

\section{DISCUSIÓN}

Para este estudio, los niños del GNE fueron seleccionados para asemejarse en peso al

Tabla 3. Datos socioeconómicos, familiares y obstétricos de las madres de los niños en estudio, nacidos en el Hospital Molas entre 2009 y 2013

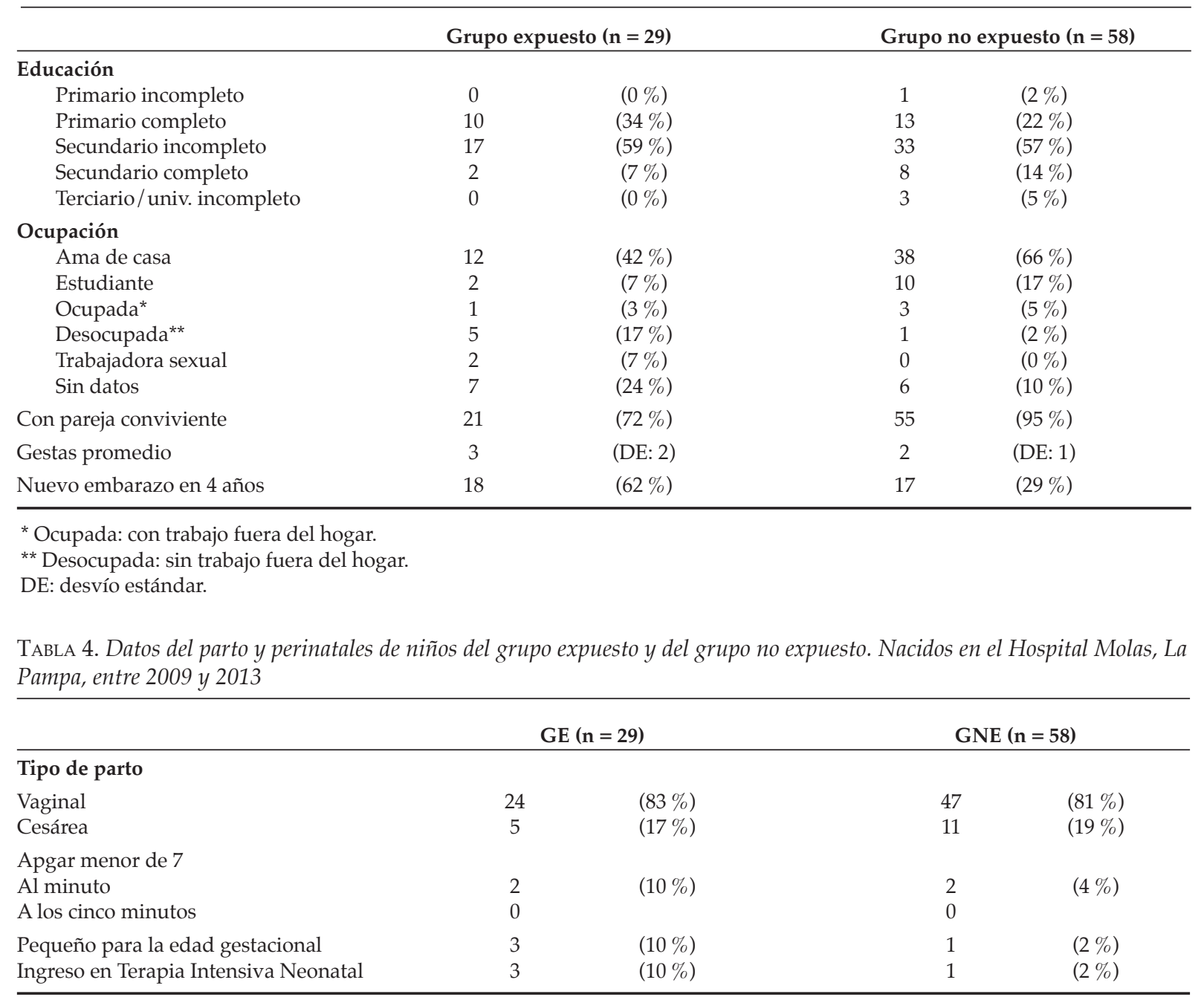

GE: grupo expuesto prenatalmente a cocaína y / o cannabis.

GNE: grupo no expuesto a esas drogas prenatalmente. 
nacer y edad gestacional a los niños del GE, por ser conocidos factores que modifican la morbimortalidad infantil y el requerimiento de controles, y que pueden afectarse por la exposición prenatal a cualquiera de las drogas consumidas por sus madres (tabaco, alcohol, cannabis o cocaína). 2,7-13,17,20,21

Además, se buscó que los niños del GNE pertenecieran a un nivel socioeconómico similar al del GE: nacidos en el mismo Servicio de Neonatología, con igual situación ante la seguridad social, con edades maternas y lugar de residencia cercanos. La existencia de un grupo de comparación doble de similar nivel socioeconómico que el GE constituye la principal fortaleza de este estudio.

Las trayectorias sanitarias de los niños, lógicamente, dependen de los adultos a cargo. De no mediar intervención o modificaciones de hábitos, el consumo de drogas y los graves factores de riesgo sociales y familiares que, por lo general, coexisten en estas familias persistirán y pasarán a formar parte del ambiente de crianza. ${ }^{12,22}$

No fue posible, en este estudio, determinar si las madres del GE continuaron consumiendo o no, aunque está descrito que el consumo posparto es muy frecuente, incluso en mujeres que lo suspendieron durante el embarazo. ${ }^{23}$

TABla 5. Comparación de motivos de consulta en los 4 primeros años de vida en niños de grupos expuesto y no expuesto. Niños nacidos en el Hospital Molas, La Pampa, entre 2009 y 2013

\begin{tabular}{|c|c|c|c|c|}
\hline & & $\begin{array}{l}\text { Grupo expuesto } \\
\qquad(\mathrm{n}=29)\end{array}$ & $\begin{array}{c}\text { Grupo no expuesto } \\
(\mathrm{n}=58)\end{array}$ & $\begin{array}{l}\text { Comparación } \\
\text { entre grupos }\end{array}$ \\
\hline \multirow[t]{2}{*}{ Controles de salud } & Media (DE) & $8(6)$ & $18(12)$ & \\
\hline & IC $95 \%$ & $6-10$ & $15-22$ & $<0,0001$ \\
\hline \multirow[t]{2}{*}{$\begin{array}{l}\text { Consultas por intercurrencia } \\
\text { en el consultorio }\end{array}$} & Media (DE) & $4(4)$ & $3(3)$ & \\
\hline & IC $95 \%$ & $3-6$ & $3-4$ & 0,338 \\
\hline \multirow[t]{2}{*}{ Consultas en Emergencias } & Media (DE) & $5(7)$ & $3(2)$ & \\
\hline & IC $95 \%$ & $3-8$ & $2-3$ & 0,0295 \\
\hline
\end{tabular}

DE: desvío estándar; IC: intervalo de confianza.

Figura 1. Motivos por los cuales requirieron 40 internaciones 19/29 niños del grupo expuesto y 23 internaciones 20/58 niños del grupo no expuesto ${ }^{* *}$ durante los 4 años del estudio***. Niños nacidos en el Hospital Molas, La Pampa, entre 2009 y 2013

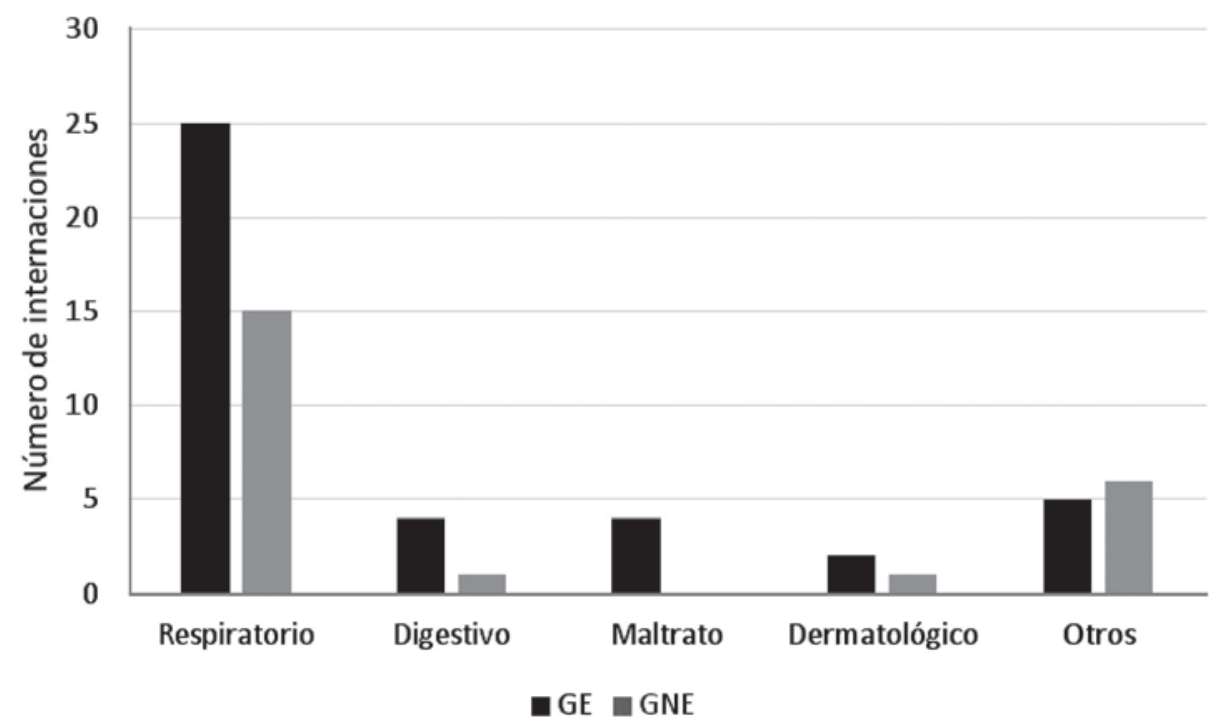

* GE: grupo expuesto prenatalmente a cocaína y/o cannabis.

** GNE: grupo no expuesto a esas drogas prenatalmente.

*** En ambos grupos, algunos niños requirieron más de una internación. 
La menor frecuencia de controles de salud de los niños del GE condice con una característica común a muchas mujeres consumidoras de drogas, que es controlar poco o nada sus embarazos. ${ }^{6-9}$ También se ha descrito que estas mujeres, con frecuencia, se niegan a participar de investigaciones, no cumplen con las citaciones o abandonan, lo que las torna muy dificultosas. Por ello, se eligió, para este estudio, un diseño de cohorte retrospectivo, en el que se utilizaron registros preexistentes, como proponían hacer otros investigadores. ${ }^{24}$

La complejidad de la problemática del consumo de drogas ilícitas en el embarazo incluye la coexistencia de graves factores de riesgo. Entre otros, las madres de los niños expuestos suelen padecer enfermedad mental y tienen antecedentes de violencia intrafamiliar en sus propias infancias y en la adultez. $3,6,8,14$

En este estudio, la violencia se muestra en los niños del GE en la existencia de judicialización por maltrato, dato presente en otros estudios, y en los cambios de hogar y de adulto a cargo., $, 13,18,19$ También se refleja en el número de muertes violentas sucedidas en algún miembro del núcleo familiar en solo 4 años. Este último dato es inédito en estudios infantiles. En Estados Unidos, se ha descrito mayor riesgo de homicidios y muertes violentas en adultos, $y$, recientemente, de homicidios de adolescentes con armas de fuego, en hogares de consumidores de drogas ilícitas..$^{25,26}$

Respecto de las internaciones, se sabe que los niños expuestos a cannabis prenatalmente ingresan con más frecuencia a Terapia Neonatal

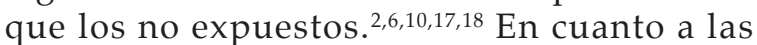
internaciones pediátricas, el $60 \%$ de los niños chilenos expuestos principalmente a cocaína (aunque también a tabaco, cannabis y alcohol, en porcentaje no aclarado) las requirieron, porcentaje similar al $66 \%$ de este estudio. La principal causa de internación en ambas investigaciones fue respiratoria. ${ }^{18}$

Asimismo, el GE se caracterizó por un elevado porcentaje de enfermedad respiratoria obstructiva crónica. La mayoría de los niños tenían código J45, "asma", pese a la dificultad de establecer el diagnóstico de asma antes de los 4 años. ${ }^{27,28}$

Los cuadros respiratorios obstructivos pueden ser provocados por diversos factores, entre otros, la exposición a drogas, en especial, si están presentes en etapas tempranas de la vida. ${ }^{29}$ Los niños del GE tuvieron madres, en su mayoría, policonsumidoras.
La exposición al tabaco es un reconocido productor de enfermedad respiratoria, y sus efectos por consumo pasivo han sido ampliamente descritos. ${ }^{29,30}$ Se ha comprobado, incluso, que la exposición prenatal al tabaco produce mayor frecuencia de obstrucción respiratoria infantil que la exposición posnatal. ${ }^{31,32}$

El cannabis provoca bronquitis crónica, y se sabe que, en su corriente principal de humo, las concentraciones de amonio, cianuros de hidrógeno y otros químicos tóxicos son más altas que en el humo de tabaco. ${ }^{33}$ Algunos autores lo han relacionado con ataques de asma y alergia, y con mayor número de hospitalizaciones. ${ }^{34}$ Sus efectos sobre el sistema respiratorio por exposición prenatal aún son inciertos, sobre todo, por la alta frecuencia de uso combinado con tabaco. 25,33

En niños de 5 años expuestos prenatalmente a tabaco y cannabis, se hallaron alteraciones en la inmunología de la mucosa respiratoria, que podrían explicar, en parte, algunos síntomas respiratorios. ${ }^{35}$

En relación con la cocaína, fue hallada con elevada frecuencia en un estudio en menores de 1 año que fueron llevados a un Servicio de Emergencias, con una correlación directa entre la concentración detectada y la presencia de síntomas respiratorios bajos. ${ }^{36}$

Respecto del alcohol, distintos estudios en animales muestran mecanismos de daño en el pulmón en desarrollo. ${ }^{37}$ Algunos hijos de consumidoras durante el embarazo asistidos en hospitales argentinos presentaron infecciones respiratorias agudas más graves que los niños sin esa exposición, pero los efectos del alcohol prenatal sobre la enfermedad obstructiva crónica fueron controvertidos. . $^{37,38}$

La limitación principal del diseño es que se requieren registros completos. En este estudio, no fue posible contar con datos de tiempo de lactancia ni de crecimiento o desarrollo adecuados.

Pese a no tener criterios de detección (y, por lo tanto, sin haberse realizado la prueba de drogas), los niños en el GNE podrían haber estado expuestos. Este factor podría afectar los resultados y disminuir las diferencias entre grupos.

No fue posible, en este estudio, determinar el rol de una u otra droga, de datos sociofamiliares adversos o de la exposición pre- o posnatal en la problemática presentada de los niños del GE. 
Pero la importancia de este estudio para la práctica diaria se relaciona con saber que los niños con exposición prenatal confirmada a cannabis y / o cocaína tienen un alto riesgo de morbimortalidad en el corto plazo. Las estrategias de abordaje y seguimiento deberían reformularse considerando que vivirán, muy probablemente, en ámbitos violentos, con frecuentes cambios de domicilio y menor concurrencia a los controles de salud que los niños no expuestos. Finalmente, esas estrategias deberían enfocarse tanto en tratar el consumo de drogas como en abordar los factores de riesgo sociofamiliares asociados.

\section{CONCLUSIONES}

Los niños expuestos a cocaína y/o cannabis prenatalmente tuvieron menor número de controles de salud y mayor frecuencia de consultas en Emergencias e Internaciones. Presentaron, además, mayor frecuencia de enfermedad pulmonar obstructiva crónica, cambios de hogar, judicialización y muertes violentas en el grupo familiar directo.

\section{REFERENCIAS}

1. Chang JC, Holland CL, Tarr JA, Rubio D, et al. Perinatal Illicit Drug and Marijuana Use: An Observational Study Examining Prevalence, Screening, and Disclosure. Am J Health Promotion. 2017; 31(1):35-42.

2. Gunn JKL, Rosales CB, Center KE, Nuñez A, et al. Prenatal exposure to cannabis and maternal and child health outcomes: a systematic review and meta-analysis. BMJ Open. 2016; 6(4):e009986.

3. Rocha PC, Alves MT, Chagas DC, Silva AA, etal. Prevalência e fatores associados ao uso de drogas ilícitas em gestantes da coorte BRISA. Cad Saúde Pública. 2016; 32(1):e00192714.

4. Forray A, Foster D. Substance use in the perinatal period. Curr Psychiatry Rep. 2015; 17(11):91.

5. Schauberger CW, Newbury EJ, Colburn JM, Al-Hamadani M. Prevalence of illicit drug use in pregnant women in Wisconsin private practice setting. Am J Obstet Gynecol. 2014; 211(3):255.e1-4.

6. Villarreal M, Ré S. Detección de drogas ilícitas en la orina en el Servicio de Neonatología, Hospital Molas, La Pampa. Arch Argent Pediatr. 2013; 111(3):232-6.

7. Magri R, Míguez H, Parodi V, Hutson J, et al. Consumo de alcohol y otras drogas en embarazadas. Arch Pediatr Urug. 2007; 78(2):122-32.

8. García García J, Campistol Mas E, López-Vilchez MÁ, Morcillo Buscato MJ, et al. Análisis del maltrato prenatal en Cataluña entre los años 2011 y 2014. An Pediatr (Barc). 2018; 88(3):150-9.

9. MartínMardomingo M,SolísSánchez G,Málaga GuerreroS, Cuadrillero Quesada C, etal. Consumo de drogas duranteel embarazo y morbilidad neonatal: cambios epidemiológicos en los últimos 10 años. An Pediatr (Barc). 2003; 58(6):574-9.

10. Bonello MR, $\mathrm{XuF}, \mathrm{LiZ}$, Burns L, et al. Mental and Behavioral Disorders Due toSubstance Abuse and Perinatal Outcomes: A Study Based on Linked Population Data in New South Wales, Australia. Int J Environ Res Public Health. 2014; 11(5):4991-5005.

11. Benevenuto SG, Domenico MD, Martins MA, Costa NS, et al. Recreational use of marijuana during pregnancy and negative gestational and fetal outcomes: An experimental study in mice. Toxicology. 2017; 376:94-101.

12. Behnke M, Smith VC; Committee on Substance Abuse; Committee on Fetus and Newborn. Prenatal substance Abuse: Short- and Long-term Effects on the Exposed Fetus. Pediatrics. 2013; 131(3):e1009-24.

13. Goel N, Beasley D, Rajkumar V, Banerjee S. Perinatal outcome of illicit substance abuse in pregnancycomparative and temporary socio-clinical profile in the UK. Eur J Pediatr. 2011; 170(2):199-205.

14. Minnes S, Lang A, Singer L. Prenatal Tobacco, Marijuana, Stimulant, and Opiate Exposure: Outcomes and Practice Implications. Addict Sci Clinl Pract. 2011; 6(1):57-70.

15. Bandstra ES, Morrow CE, Mansoor E, Accornero VH. Prenatal drug exposure: Infant and toddler outcomes. J Addict Dis. 2010; 29(2):245-58.

16. Lamy S, Laqueille X, Thibaut F. Conséquences potentielles de la consommation de tabac, de cannabis et de cocaïne par la femme enceinte sur la grossesse, le nouveau-né et l'enfant: revue de littérature. Encephale. 2015; 41(Suppl 1):S13-20

17. Hayatbakhsh M, Flenady VJ, Gibbons KS, Kingsbury AM, et al. Birth outcomes associated with cannabis use before and during pregnancy. Pediatr Res. 2012; 71(2):215-9.

18. Mena RM, Navarrete MP, Corvalán VS, Bedregal GP. Drogadicción embriofetal por abuso de pasta base de cocaína durante el embarazo. Rev Méd Chile. 2000; 128(10):1093-100.

19. Jaudes PK,EkwoE, Van VoorhisJ. Association of drug abuse and child abuse. Child Abuse Negl. 1995; 19(9):1065-75.

20. Ostrea EM,Ostrea AR, SimpsonPM. Mortality within the first 2 years in infants exposed to cocaine, opiate, or cannabinoid during gestation. Pediatrics. 1997; 100(1):79-83.

21. Coleman-CowgerVH,OgaE, PetersEN, MarkK.Prevalence and associated birth outcomes of co-use of Cannabis and tobacco cigarettes during pregnancy. Neurotoxicol Teratol. 2018; 68:84-90.

22. Serino D, Peterson BS, Rosen TS. Psychological Functioning of Women Taking Illicit Drugs during Pregnancy and the Growth and Development of Their Offspring in Early Childhood. J Dual Diagn. 2018; 14(3):158-70.

23. Forray A, Merry B, LinH, RugerJP, etal. Perinatal Substance Use: A Prospective Evaluation of Abstinence and Relapse. Drug Alcohol Depend. 2015; 150:147-55.

24. Gabrhelík R, Nechanská B, Mravčík V, Skurtveit S, et al. A unique opportunity to Study Short- and Long-Term Consequences in Children Prenatally Exposed to Illicit Drugs and Opioid Maintenance Treatment Using Czech and Scandinavian Registers. Cent Eur J Public Health. 2016; 24(3):248-51.

25. Rivara FP, Mueller BA, Somes G, Mendoza CT, et al. Alcohol and Illicit Drug Abuse and the Risk of Violent Death in the Home. JAMA. 1997; 278(7):569-75.

26. HohlBC, Wiley S, Wiebe DJ, Culyba AJ, et al. Association of Drug and Alcohol Use With Adolescent Firearm Homicide at Individual, Family, and Neighborhood Levels. JAMA Intern Med. 2017; 177(3):317-24.

27. Pedersen SE, Hurd SS, Lemanske RF Jr, Becker A, et al. Global strategy for the diagnosis and management of asthma in children 5 years and younger. Pediatr Pulmonol. 2011; 46(1):1-17.

28. Klein M. Critique of GINA global strategy. Pediatr Pulmonol. 2012; 47(1):99-100.

29. Carraro S, Scheltema N, Bont L, Baraldi E. Early-life origins of chronic respiratory diseases: understanding and promoting healthy ageing. Eur Respir J. 2014; 44(6):1682-96.

30. McEvoy CT, Spindel ER. Pulmonary effects of maternal 
smoking on the fetus and child: effects on lung development, respiratory morbidities, and life long lung health. Paediatr Respir Rev. 2016; 21:27-33.

31. Vardavas CI, Hohmann C, Patelarou E, Martinez D, et al. The independent role of prenatal and postnatal exposure to active and passive smoking on the development of early wheeze in children. Eur Respir J. 2016; 48(1):115-24.

32. Neuman A, Hohmann C, Orsini N, Pershagen G, et al. Maternal smoking in pregnancy and asthma in preschool children: a pooled analysis of eight birth cohorts. Am J Respir Crit Care Med. 2012; 186(10):1037-43.

33. Kreuter M, Nowak D, Rüther T, Hoch E, et al. Cannabis-Positionspapier der Deutschen Gesellschaft für Pneumologie und Beatmungsmedizin e.V. (DGP). Pneumologie. 2016; 70(2):87-97.

34. Chatkin JM, Zani-Silva L, Ferreira I, Zamel N. Cannabis-
Associated Asthma and Allergies. Clin Rev Allergy Immunol. 2017; 56(2):196-206.

35. Molnar DS, Granger DA, Shisler S, Eiden RD. Prenatal and postnatal cigarette and cannabis exposure: Effects on Secretory Immunoglobulin A in early childhood. Neurotoxicol Teratol. 2018; 67:31-6.

36. Lustbader AS, Mayes LC, McGee BA, Jatlow P, et al. Incidence of passive exposure to crack/ cocaine and clinical findings in infants seen in an outpatient service. Pediatrics. 1998; 102(1):e5.

37. Gauthier TW, Brown LA. In utero alcohol effects on foetal, neonatal and childhood lung disease. Paediatr Respir Rev. 2016; 21:34-7.

38. Libster R, Ferolla FM, Hijano DR, Acosta PL, et al. Alcohol during pregnancy worsens acute respiratory infections in children. Acta Paediatr. 2015; 104:e494-9.

\section{Archivos hace 75 años}

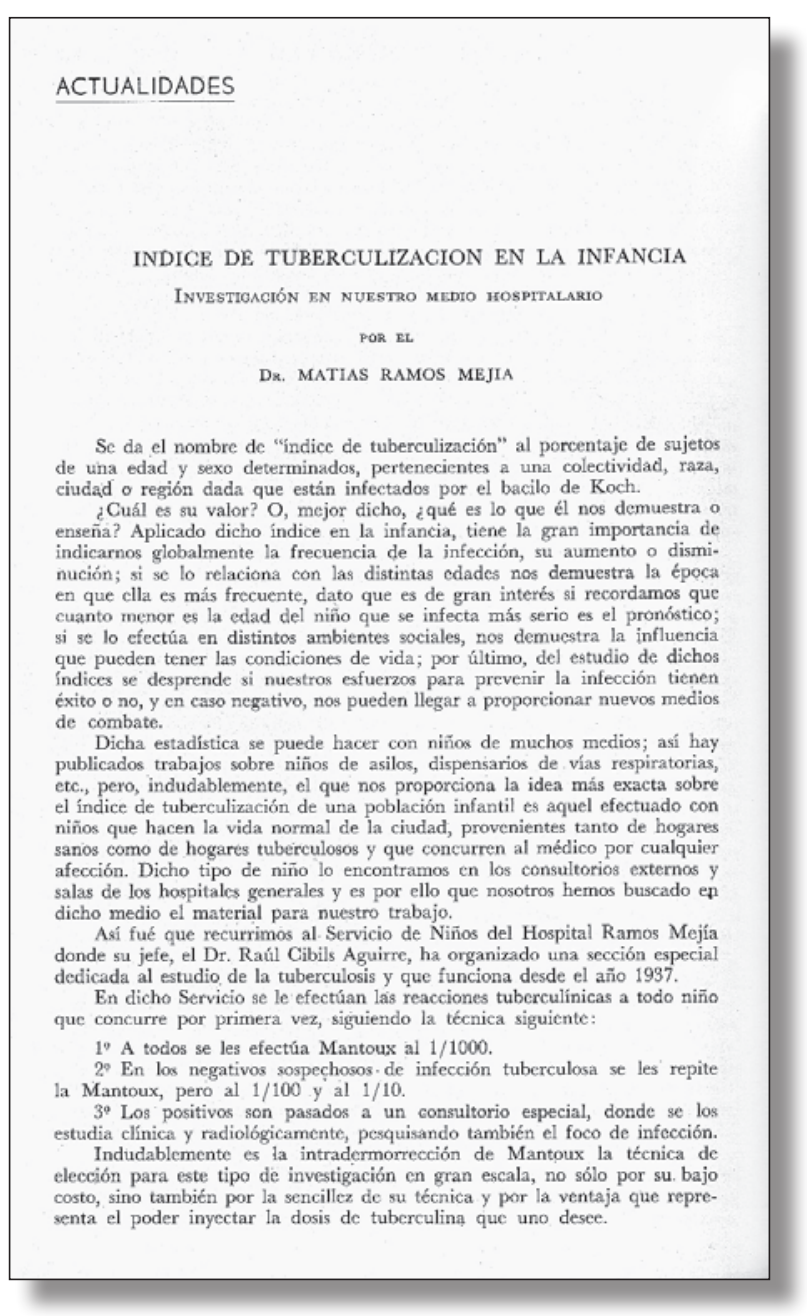

El texto completo se encuentra disponible en la versión electrónica de este número. 